\title{
Amniotic Sac Herniation Through a Prior Cornual Scar in The Third Trimester
}

\author{
Antonio F. Saad, MD ${ }^{1}$ Maged M. Costantine, MD ${ }^{1} \quad$ George Saade, MD ${ }^{1} \quad$ Michel Makhlouf, MD, PHD ${ }^{1}$ \\ ${ }^{1}$ Division of Maternal-Fetal Medicine, Department of Obstetrics and \\ Gynecology, The University of Texas Medical Branch, Galveston, \\ Address for correspondence Antonio F. Saad, MD, 301 University \\ Boulevard, Galveston, TX 77555 (e-mail: afsaad@utmb.edu).
} Texas

Am J Perinatol Rep 2015;5:e132-e135.

\begin{abstract}
Keywords

- cornual pregnancy

- spontaneous uterine rupture

- uterine surgery

Introduction Uterine rupture occurs in less than $0.1 \%$ of pregnancies. This complication can be detrimental to mother and fetus if not detected and managed in a timely manner. We report an unusual presentation of uterine scar rupture that was diagnosed on ultrasound in a completely stable patient with reassuring fetal status.

Case Report A 24-year-old Gravida 5, Para 3 with history of cornual resection for ectopic pregnancy and two previous uterine ruptures presented at 30 weeks' gestation with worsening abdominal pain. Ultrasound identified herniation of the amniotic sac with fetal parts. The patient underwent cesarean delivery and cornual defect repair. Conclusion Close observation and early delivery remain vital to the patient's management.
\end{abstract}

Uterine rupture can be a catastrophic event that most obstetricians would prefer not to encounter. Mortality and morbidity that accompany this event can be detrimental to both the mother and fetus. In the United States, it occurs in less than $0.1 \%$ of pregnancies. A major risk factor is a uterine scar from previous procedures such as cesarean delivery, active segment hysterotomy, myomectomy, or transcornual disruption. Other contributing factors are macrosomia, abnormal placentation, uterine anatomic anomalies, grand multiparity, high-dose oxytocin, and obstetric trauma. Most uterine ruptures occur late in the third trimester, during trial of labor, but there are occasional reports of a uterine scar rupture and exteriorization of uterine contents in a gravid uterus in a patient without signs or symptoms. ${ }^{1-4}$ We report an unusual presentation of uterine scar rupture that was diagnosed on ultrasound in a completely stable patient with reassuring fetal status.

\section{Case Report}

A 24-year-old (Gravida 5, Para 3) at 30 weeks' gestation presented to labor and delivery triage on September 9, 2013 with complaints of abdominal pain of 2 weeks' duration aggravated over the past 2 days. Her history was significant for laparoscopic resection of a right cornual ectopic pregnancy in 2009. At that time, the cornual defect was overlaid with Nu-Knit Surgicel absorbable Hemostat (Ethicon, CA). In 2011, patient had a cesarean delivery at 39 weeks' of gestation for fetal intolerance of labor. On entering the abdomen copious blood was found and fetal extremity with intact membranes was noted extending through a corneal uterine rupture. The fetus was delivered through a low transverse incision; the cornual defect was closed with Vicryl in the three layers. In 2012, the patient presented with placental abruption at 29 weeks of gestation and underwent an emergent cesarean delivery. Findings at the time of delivery revealed right cornual uterine rupture with portions of the placenta and fetus located outside the uterus with intact membranes. Estimated blood loss was $2,500 \mathrm{~mL}$ and patient received a total of 2 units of packed red blood cells. The infants from both pregnancies are currently alive and well. These two pregnancies and associated postpartum care were not managed at our facility but at a local, nontertiary care hospital. At the time of presentation to triage in this pregnancy, the patient reported the pain was generalized over the abdominal area, constant, and nonradiating. Vital signs were within normal limits. The received

April 14, 2014

accepted after revision

May 27, 2014

published online

April 27, 2015
DOI http://dx.doi.org/ 10.1055/s-0035-1549296. ISSN 2157-7005.
Copyright $\odot 2015$ by Thieme Medical Publishers, Inc., 333 Seventh Avenue, New York, NY 10001, USA. Tel: +1(212) 584-4662.

\section{License terms}

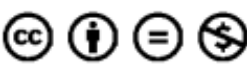




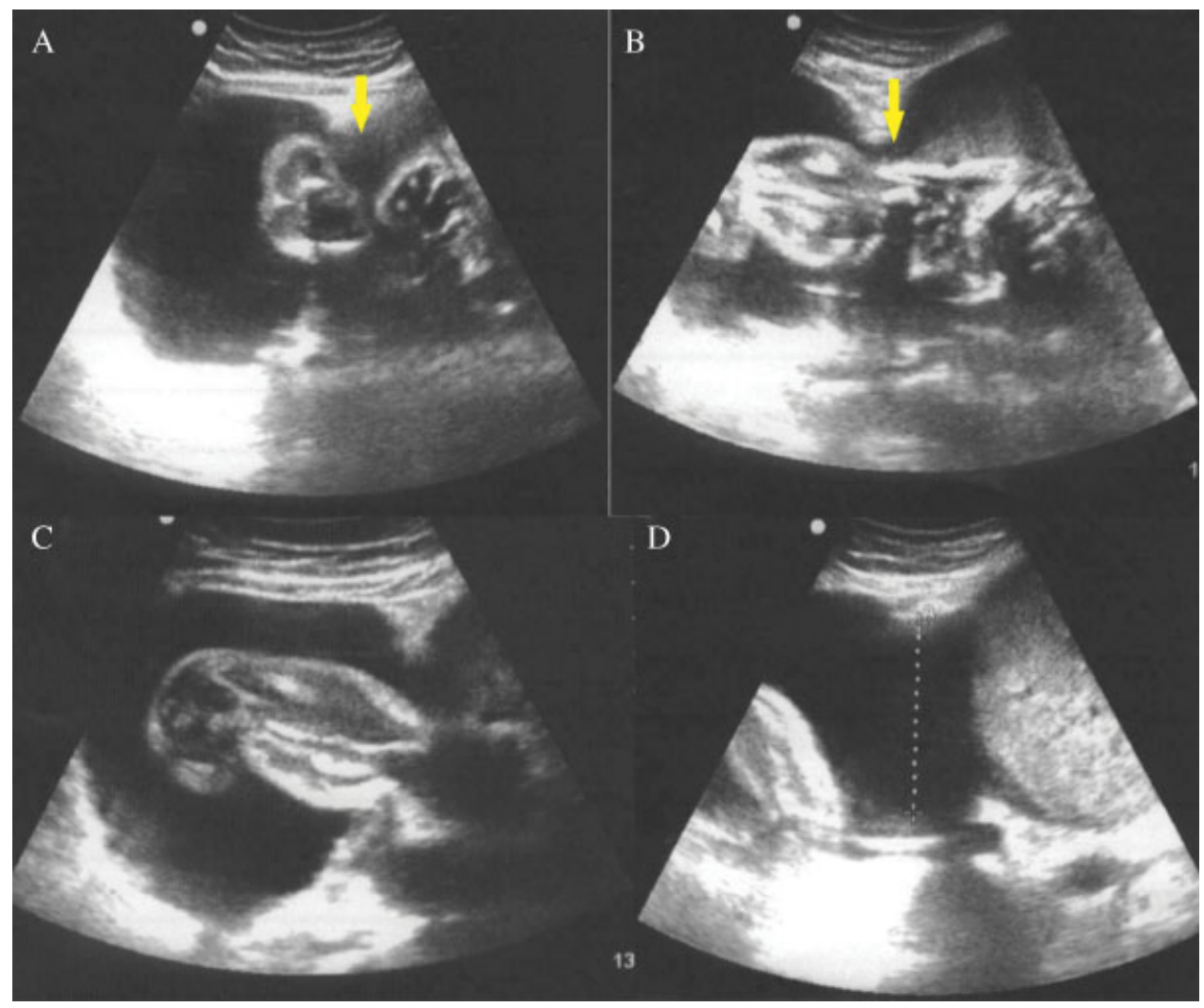

Fig. 1 (A-D) Uterine defect noted in right cornual area (arrows) with herniation of the amniotic membrane and right fetal low-extremity intact. In $B$, the thigh appears swollen.

examination revealed no evidence of acute abdomen or peritonitis, negative Murphy and McBurney signs. Fetal heart rate monitoring was reassuring and appropriate for gestational age with minimal uterine irritability detected on the tocometer. A bedside ultrasound examination ( $\boldsymbol{- \text { Fig. }}$ 1A-D) revealed a uterine defect in the right cornual area with herniation of the amniotic membrane and right fetal lower extremity. The thigh appeared swollen, but the movement of the lower extremity was observed. All of the amniotic fluid was located in the herniated sac and virtually none was seen in the uterus. The patient was taken for a cesarean delivery. Operative findings (see - Fig. 2A-C) confirmed the diagnosis
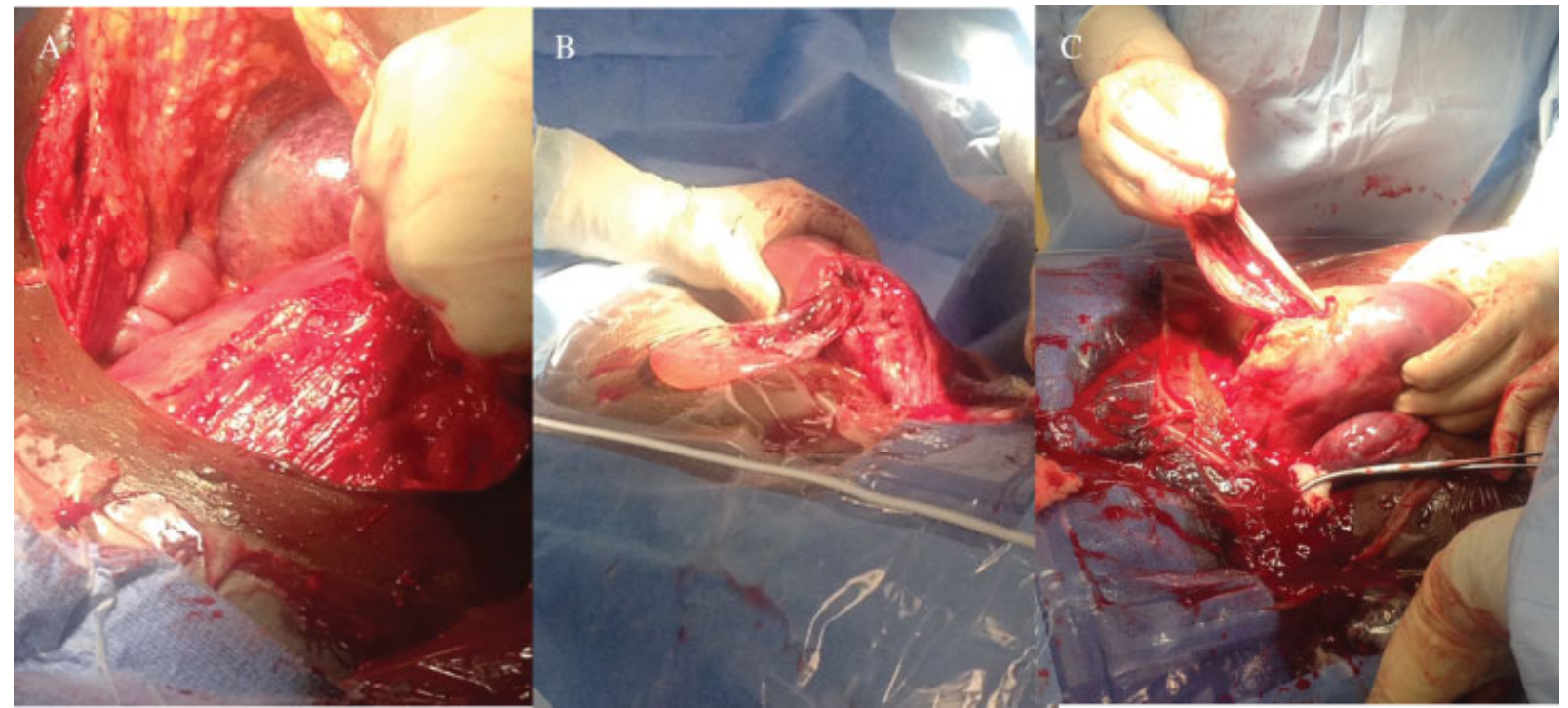

Fig. 2 (A-C) Right corneal uterine scar rupture noted after fetus was delivered though a low transverse cesarean delivery. 
of right cornual uterine scar rupture. The fetus was delivered through a low transverse uterine incision with no complications and appropriate blood loss. The male fetus weighed $1,680 \mathrm{~g}$ and had an umbilical artery cord $\mathrm{pH}$ of 7.28 and a base deficit of 3.1. Apgar scores were noted to be 4 (1 minute), 8 (5 minutes). Both low transverse incision and uterine rupture sites were closed in a double-layer fashion with a 0-monocryl suture. Transection of the left fallopian tube was performed per patient request and prior consent. Postoperative recovery of the mother was uneventful with routine care. The infant was transferred to the neonatal intensive care unit. The examination of right lower extremity revealed no deformities, normal range of motion, mild ecchymosis, and moderate edema.

\section{Comment}

Uterine rupture consists of a complete defect of all uterine layers, including the serosa. It is an emergent and life-threatening condition for both the mother and fetus. Most patients are at risk of massive hemorrhage, hysterectomy, bladder laceration, and perinatal death or neurological injury from hypoxia or acidosis. The perinatal death rate associated with uterine rupture is high (5-6\%). ${ }^{1}$ Major risk factors for uterine rupture include classical cesarean delivery (risk of rupture ranges from 1 to $12 \%$ ), any transcornual disruption, prior cesarean deliveries (highest in classical uterine incision), and prior uterine rupture. ${ }^{2}$ Most uterine ruptures occur in the inpatient setting during trial of labor after cesarean delivery. ${ }^{3-5}$ Case reports have been published of spontaneous uterine rupture in primigravidas. ${ }^{6}$ The classic presentation of uterine rupture is that of hemorrhagic shock, fetal bradycardia, and loss of fetal station. The fetus is often compromised with neurological impairment because of the hypoxia and with long-term disability. ${ }^{7}$

We report an unusual case of a patient with a significant history of cornual disruption secondary to ectopic pregnancy with previous two uterine ruptures at the prior site of cornual repair. Her early second trimester ultrasound revealed a normal intrauterine pregnancy with a normal size uterus. Maternal fetal medicine specialist recommended close observation throughout the pregnancy, and early delivery depending on signs, symptoms, and ultrasound findings. A firm delivery date was not set, however, it was expected that the patient will deliver preterm considering her history. In addition, the patient was counseled to present to the office or triage if she had moderate or severe or persistent abdominal pain. She was also counseled about tubal ligation because of her poor obstetrical history and agreed to sterilization. Counseling after any uterine rupture should include discouragement of subsequent pregnancies and tubal ligation should be offered. It was unknown in this case whether this counseling was offered during her past pregnancies. At a presentation in triage, the patient was stable, did not appear in distress or severe pain, had normal laboratory work up, and reassuring fetal status. Ultrasound examination revealed uterine scar rupture with partial extrusion of the fetus with an intact amniotic membrane. This is not a classical presentation of a catastrophic uterine rupture.
It is therefore important for physicians to recognize that stable vitals and examinations do not exclude the possibility of uterine rupture. Heightened awareness is imperative in order to not miss such a diagnosis. Patients with prior uterine rupture are at high risk for recurrence. If the prior uterine rupture is confined to the lower uterine segment, the recurrence rate of rupture in labor is $6 \%{ }^{7}$ On the contrary, if the scar includes the active segment of the uterus, that rate may be as high as $32 \%{ }^{8}$ Hence, antenatal close follow-up is critical; encounters should be scheduled in short intervals. Patient education should focus on early uterine rupture warnings with a lower threshold to present for evaluation than a normal pregnancy. Although the optimal frequency of ultrasound examinations in such cases is unknown, ultrasound remains an indispensable tool for assessment and follow-up. Spontaneous labor remains unpredictable and can happen before 39 weeks of gestation; therefore in these cases, an early delivery after administration of antenatal corticosteroids or confirmation of fetal lung maturity must be considered. Recent evidence indicates that the technique of uterine closure plays an important role in uterine healing. Single layer uterine closure with the inclusion of the endometrium has been associated with increased rates of uterine defect, dehiscence, and rupture in consecutive pregnancies, ${ }^{8}$ and thus, double layer closure with longer lasting suture is recommended.

In our case, the patient elected to have permanent sterilization. However, for other patients who desire future fertility, management may be challenging given the risk of rupture. Gestational surrogate carrier with in vitro fertilization is a safer alternative but may not be achievable for patients with limited financial or health access resources.

\section{Financial Disclosure}

Dr. Saade is the editor-in-chief of the submitted journal.

We attest of obtaining written consent from the patient permitting publication/research of this case.

\section{Condensation}

Spontaneous uterine rupture after uterine surgery can be catastrophic. History, physical examination, and close antenatal follow-up is crucial even in the absence of classical clues.

\section{References}

1 Turner MJ. Uterine rupture. Best Pract Res Clin Obstet Gynaecol 2002;16(1):69-79

2 Leung AS, Farmer RM, Leung EK, Medearis AL, Paul RH. Risk factors associated with uterine rupture during trial of labor after cesarean delivery: a case-control study. Am J Obstet Gynecol 1993;168(5): 1358-1363

3 Lazarus EJ. Early rupture of the gravid uterus. Am J Obstet Gynecol 1978;132(2):224

4 Iddenden DA, Nuttall ID. Early spontaneous rupture of the gravid uterus. Am J Obstet Gynecol 1983;147(8):971-972 
5 Auamkul S. Rupture of gravid uterus after cornual resection. A case report. J Reprod Med 1970;5(5):218-220

6 Walsh CA, O'Sullivan RJ, Foley ME. Unexplained prelabor uterine rupture in a term primigravida. Obstet Gynecol 2006;108(3 Pt 2): $725-727$
7 Ritchie EH. Pregnancy after rupture of the pregnant uterus. A report of 36 pregnancies and a study of cases reported since 1932. J Obstet Gynaecol Br Commonw 1971;78(7):642-648

8 Bujold $\mathrm{E}$. The optimal uterine closure technique during cesarean. N Am J Med Sci 2012;4(8):362-363 